\title{
Evaluación de la productividad primaria en un gradiente de precipitación, en un pastizal patagónico
}

\author{
Evaluation of primary productivity in a precipitation gradient, in a Patagonian \\ grassland
}

\author{
Jesabel Schön, Daniela Ferrante, Gabriel Oliva \\ jesabel.schon@gmail.com
}

Universidad Nacional de la Patagonia Austral

Av. Piloto “Lero“ Rivera y Av. Gdor. Gregores - Río Gallegos - Santa Cruz - Argentina

Recibido: 06/02/2020. Aceptado: 11/09/2020

\section{RESUMEN}

La disponibilidad de agua es el factor principal que determina la producción primaria de los pastizales en zonas áridas y semiáridas. En noviembre de 2018, en el sur de Patagonia, Estepa Magallánica Seca, se instaló un ensayo de disponibilidad hídrica, con el fin de evaluar la productividad primaria aérea neta (PPAN) de gramíneas altas y bajas frente a distintos niveles de ingreso de agua. Los tratamientos fueron: sequía con interceptores de lluvia (reciben 50\% menos de la precipitación anual), tratamiento húmedo $(60 \mathrm{~mm}$ de agua agregados en el periodo seco) y testigo, tres parcelas por tratamiento excluidas del pastoreo. En estas parcelas se instalaron sensores de potencial hídrico de suelo a $10 \mathrm{~cm}$ de profundidad y un pluviómetro conectados a un logger con frecuencia de medición de una hora. La productividad anual se estimó por cosecha de biomasa en marcos de $0,6 \mathrm{~m}^{2}$ y separación de material en verde y seco, los cortes se realizaron al final de la estación de crecimiento (marzo 2019). Los tratamientos de disponibilidad hídrica no afectaron la PPAN, pero si mostraron diferencias en los valores de humedad de suelo, el suelo en el tratamiento de riego estuvo más húmedo. Posiblemente se requiera de un periodo más extenso para que la sequía se manifieste en el suelo y en la vegetación.

Palabras clave: sequía; gramíneas; interceptores de lluvias.

\begin{abstract}
Water is the main factor controlling the primary production in arid and semi-arid grasslands. A rainfall intercept experiment was installed in southern Patagonia in November 2018. In this trial we evaluated net primary aerial productivity (PPAN) of tussock and short grasses with different rainfall levels. The treatments were: drought with rain shelters (that received $50 \%$ of the annual precipitation), wet treatment ( $60 \mathrm{~mm}$ of water added in the dry period) and control, that consisted in three plots per treatment. All plots were excluded from grazing, and were provided with soil water potential sensors at a $10 \mathrm{~cm}$ soil depth and connected to a logger together with a single rain gauge for the site. The measurement frequency was one hour. Annual productivity was estimated by harvesting biomass in $0.6 \mathrm{~m}^{2}$ plot and we separated biomass in green and dry. Harvests were made at the end of the growing season (March 2019). The treatments did not affect the PPAN, but showed differences in the soil water
\end{abstract}


potential, as soil in the irrigation treatment was more humid. It is possible that longer periods are required for drought to manifest in the soil water potential and in vegetation productivity.

Key words: drought; grasses; rain interceptors.

\section{INTRODUCCIÓN}

En los ecosistemas áridos, la precipitación es el principal factor de control de los procesos biológicos, es altamente variable entre años y ocurre en eventos poco frecuentes (Noy Meir, 1973). La productividad primaria aérea neta (PPAN) responde linealmente a la precipitación en modelos espaciales (Sala et al., 1988), pero en los modelos temporales son otros los controles ambientales que condicionan la dinámica de la PPAN (Yadhjian y Sala, 2002). Uno de ellos, especialmente en ambiente fríos, es la temperatura que determina el inicio de la temporada de crecimiento. La temperatura media del mes de julio es la que mejor explica el inicio del crecimiento. Si es $<0{ }^{\circ} \mathrm{C}$ el crecimiento inicia más tarde en el mes de octubre, mientras si es $>0^{\circ} \mathrm{C}$ inicia antes, a mediados de agosto. El modelo propone una relación cuadrática en la cual la fecha de inicio de la temporada de crecimiento (Día Juliano) $=-1,1$ $\left(\mathrm{T}^{\circ} \text { Julio }\right)^{2}-7,1\left(\mathrm{~T}^{\circ}\right.$ Julio $)+278 ; \mathrm{R}^{2}=0.57 ; \mathrm{P}<0.001$ (Jobbaggy et al., 2002) .

En pastizales áridos del mundo se han utilizado ensayos de intercepción de lluvias para generar gradientes de precipitación mediante cambios en la cantidad total, en la distribución estacional o en la frecuencia y el tamaño de los pulsos en los que la lluvia ingresa al ecosistema (Yahdjian y Sala, 2011). Las manipulaciones ayudan a comprender las consecuencias ecológicas del cambio de las precipitaciones sobre diferentes variables, principalmente la PPAN de los pastizales (Yahdjian y Sala, 2006).

Para simular eventos de sequía se construye una estructura de ubicación fija, con un techo que consiste en canaletas de acrílico transparente que interfieren en diferentes proporciones, según la cantidad de canaletas, el ingreso de agua de lluvia mientras que afecta mínimamente otras variables ambientales como la temperatura. Esta intervención puede producir contenidos de agua significativamente menor $(\mathrm{P}<0.05)$ hasta $30 \mathrm{~cm}$ de profundidad (Yadjhian y Sala, 2002). En el sur de Patagonia, la vegetación dominante son las gramíneas. En el suelo existen periodos secos en el verano, que se pueden prolongar hasta el otoño dependiendo de la temperatura y la precipitación. Durante el invierno y principios de la primavera el perfil (0-60 $\mathrm{cm})$ se encuentran cerca de la capacidad de campo $(\approx 0 \mathrm{MPa})$ (Ferrante et al., 2014). Los distintos grupos funcionales (gramíneas altas, gramíneas cortas, subarbustos) responden diferencialmente a las variaciones en la disponibilidad hídrica en función de la distribución de raíces en el perfil del suelo (Sala et al., 1997). Las gramíneas cortas tienen raíces más superficiales, en comparación con gramíneas medianas y leñosas y exploran un volumen menor de suelo (Ferrante, 2011).

En este trabajo se plantea la siguiente hipótesis: el gradiente de lluvia generará cambios en la disponibilidad hídrica superficial del suelo y esto impactará mayormente sobre la PPAN del grupo funcional con raíces más superficiales (gramíneas cortas).

Los objetivos del mismo son nombrados a continuación:

1. Cuantificar las variaciones de la PPAN de dos tipos funcionales de los pastizales del sur patagónico: gramíneas altas y gramíneas cortas en un ensayo manipulativo de precipitación. 
2. Determinar el efecto de la manipulación de lluvia sobre el potencial hídrico de suelo superficial $(0-10 \mathrm{~cm})$.

Este tipo de ensayos permite reemplazar el componente temporal por el espacial, ya que en un mismo año se evalúa la PPAN con diferentes niveles de lluvia.

\section{MATERIALES Y METODOS}

$\underline{\text { Descripción del sitio de estudio }}$

El sitio de estudio se encuentra a unos $100 \mathrm{~km}$ al oeste de la localidad de Río Gallegos, Santa Cruz, Argentina, en el Campo experimental "Potrok aike" de INTA Santa Cruz (-51.91603 LS -70.4074444 ${ }^{\circ}$ LO). El área ecológica corresponde a la Estepa Magallánica Seca, la cual presenta una precipitación anual de $200 \mathrm{~mm}$ que, si bien se distribuye a lo largo de todo el año, presenta un máximo estival. El promedio anual de temperatura varía de 6 a $7^{\circ} \mathrm{C}$. (Oliva et al., 2001). Los vientos son predominantes del suroeste y presentan una velocidad media anual de $27 \mathrm{~km} / \mathrm{h}$ (Ferrante, 2011).

La vegetación corresponde a la Unidad Fisonómica Florística Estepa Graminosa Xérica, representada por Festuca gracillima con Nardophyllum bryoides. La especie dominante, $F$ gracillima, alcanza una cobertura de $40 \%$ y entre sus matas se presenta un estrato de pequeñas gramíneas, graminoides y hierbas: Rytidosperma virescens, Poa speciformis, Carex andina, Viola maculata, Calceolaria uniflora, Azorella fueguina, entre otras, siendo Poa spiciformis (ex Poa dusenii) la de mayor cobertura en este estrato (León et al., 1998).

Descripción del ensayo

Se establecieron nueve parcelas de $2 \times 2 \mathrm{~m}\left(4 \mathrm{~m}^{2}\right)$, las cuales fueron divididas en tres tratamientos de disponibilidad hídrica, como se detalla a continuación:

- $50 \%$ de intercepción de lluvia (nueve canaletas de acrílico que obstruyen el 50\% de la superficie de la parcela, generando un $50 \%$ de intercepción de las lluvias).

- Tratamiento de riego (50\% más de lluvia en verano). Adición de un 50\% del volumen de lluvia $(60 \mathrm{~mm})$ distribuidos en eventos de $10 \mathrm{~mm}$. Las fechas de riego fueron las siguientes: 13/12/18, 20/12/18, 10/01/19, 21/01/19, 04/02/19 y 15/02/19. El riego se realizó con regadera, manualmente.

- Sin intervención (testigo).

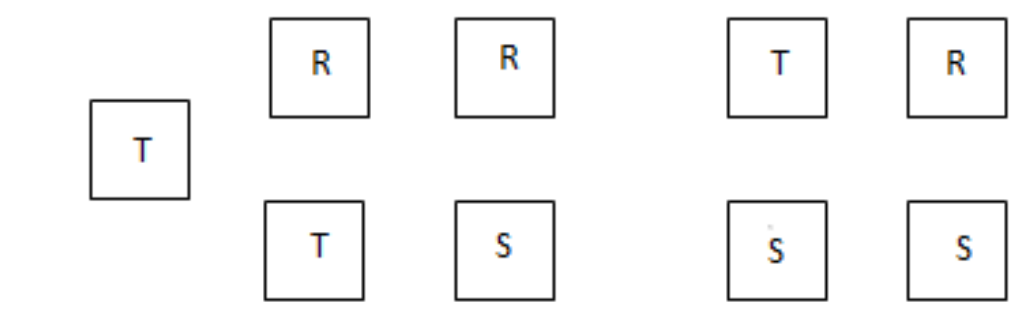

$\mathrm{T}$ : tratamiento testigo R: tratamiento riego S: tratamiento sequia

Figura 1. Croquis de la ubicación de las parcelas y los distintos tratamientos. 


\section{$\underline{\text { Variables climáticas }}$}

Se obtuvieron datos de precipitación y temperatura del aire de la Estación Meteorológica Potrok Aike, para caracterizar la distribución anual de las precipitaciones y evaluar si el año correspondiente al ensayo fue un año húmedo, seco o normal. Además, se calculó el inicio de la temporada de crecimiento de Potrok Aike mediante la fórmula propuesta por Jobbaggy et al., 2002: Fecha de inicio de crecimiento (día Juliano) $=-1,1\left(\mathrm{~T}^{\circ} \mathrm{Julio}^{2}-7,1\left(\mathrm{~T}^{\circ} \mathrm{Julio}^{2}\right)+\right.$ 278. La misma requiere la temperatura del mes de Julio para su cálculo.

Asimismo, se instaló un pluviómetro (Decagon device ECRN-100) conectado a un data lagger con mediciones horarias en el ensayo y un sensor (Decagon device 6HS) de potencial hídrico y temperatura del suelo, con frecuencia de registro horaria, en cada parcela. Los datos se presentan mensualmente como el resultado promedio diario de potencial hídrico de cada parcela.

\section{$\underline{\text { Productividad primaria }}$}

En cada parcela se determinó la productividad primaria neta aérea anual al final de la estación de crecimiento, marzo 2019, como se sugiere para ambientes áridos (Sala y Austin 2000). Se cosechó por separado la biomasa de gramíneas altas (coirones) y bajas (intercoironal), en un marco rectangular de $0.6 \mathrm{~m}^{2}$, antes de cosechar se obtuvo una fotografía de cada uno para obtener la cobertura por forma de vida. La biomasa fue secada en horno por 48 horas a $60^{\circ} \mathrm{C}$. La biomasa total de cada estrato (coirones e intercoirones) de cada parcela se dividió en cuatro fracciones, un cuarto se separó en verde y seco a fin de estimar la productividad primaria. Finalmente se pesaron las muestras con balanza analítica. Se consideró la fracción VERDE como la biomasa producida en el año, esta fracción es considerada la productividad anual en gramíneas.

Para estimar la cobertura de los distintos estratos (coirones e intercoironal) se utilizó el programa "Image j" a fin de ponderar o utilizar como factor de corrección para el cálculo de la productividad. Cada fotografía se dimensiono en centímetros conociendo el tamaño del marco. Se digitalizaron polígonos identificando aquellos correspondientes a coirones y gramíneas cortas y se sumaron las áreas de cada uno de ellos. La cobertura de cada forma de vida se obtuvo como la sumatoria $\left(\mathrm{cm}^{2}\right)$ de los polígonos sobre el área total del marco.

\section{Productividad primaria aérea neta (PPAN)}

La PPAN de cada forma de vida se obtuvo como: la biomasa total cosechada en cada parcela por la proporción de verde, por la cobertura relativa de esa forma de vida en el marco $\left(0,6 \mathrm{~m}^{2}\right)$. El resultado final se expresó en $\mathrm{g} / \mathrm{m}^{2}$.

\section{$\underline{\text { Análisis estadístico }}$}

Para analizar los efectos de los tratamientos, los resultados obtenidos se analizaron estadísticamente con análisis de varianza con el software Infostat. Se utilizó ANOVA de un factor (disponibilidad hídrica), Test de Duncan con nivel de significancia de 0,05. Se analizó por separado la productividad de cada forma de vida y en el caso de potencial hídrico el análisis se realizó por fecha desde diciembre a marzo. 


\section{RESULTADOS Y DISCUSION}

\section{$\underline{\text { Variables climáticas }}$}

\section{Precipitaciones}

La precipitación anual del año 2018 fue de 196 mm, correspondiente a un año normal. Los registros máximos fueron en abril y mayo, siendo los valores 33 y 30, $6 \mathrm{~mm}$ respectivamente. (Figura 2).

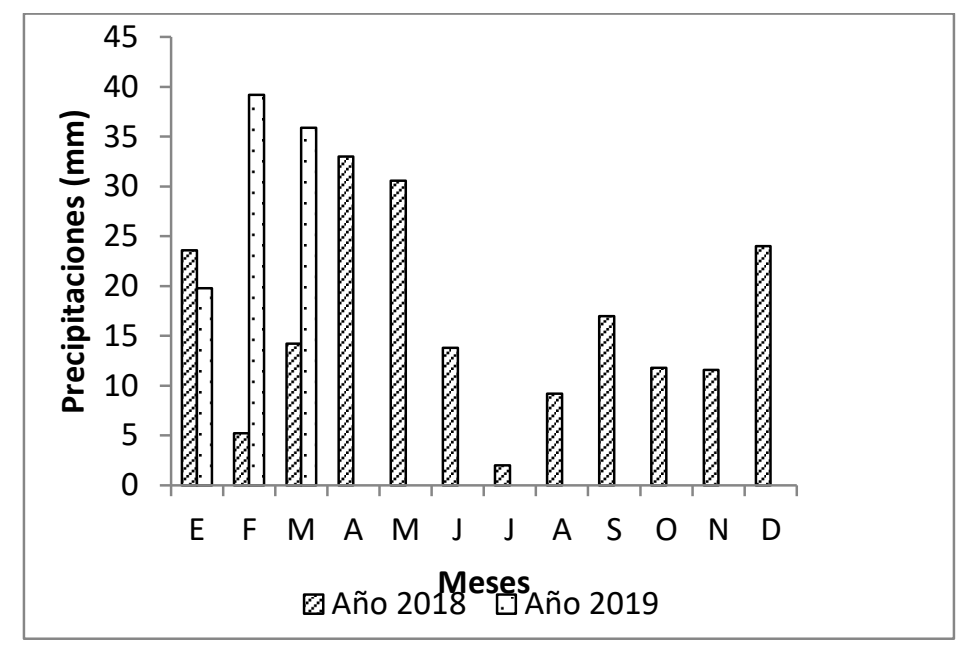

Figura 2. Precipitaciones registradas en Potrok Aike en el año 2018 y verano de 2019.

En verano de 2019, los registros fueron muy superiores a los del año 2018, llovió ocho veces más en el mes de febrero y dos veces más en el mes de marzo.

\section{Temperatura mes de julio}

La temperatura del mes de julio registrada por la Estación Meteorológica Potrok aike fue de $-1{ }^{\circ} \mathrm{C}$. Con dicho valor se obtuvo que el inicio de la estación de crecimiento comenzó el día Juliano (284), que equivale al 11 de octubre del 2018.

\section{Potencial hídrico}

Los valores de potencial hídrico de diciembre y enero entre los tratamientos riego y testigo mostraron diferencias significativas $(\mathrm{p}<0.05)$. El potencial hídrico del suelo en Diciembre en testigo fue de $-35,86 \mathrm{MPa}$, que indica que el suelo estuvo seco, mientras que en el de riego fue de $-4,33 \mathrm{MPa}$ (suelo más húmedo). El tratamiento de sequía presentó valores intermedios y no se diferenció de los restantes tratamientos. En el mes de enero el perfil del suelo continuó secándose. Los valores de potencial hídrico se duplicaron en el testigo $(-68,1 \mathrm{MPa})$ y en el de riego alcanzaron valores de $-23,8 \mathrm{MPa}$, aunque las diferencias estadísticas se mantuvieron.

En febrero el perfil comienza a humedecerse y la humedad de suelo fue similar entre tratamientos, en marzo el perfil se saturó con valores cercanos a capacidad de campo (Figura 3). 


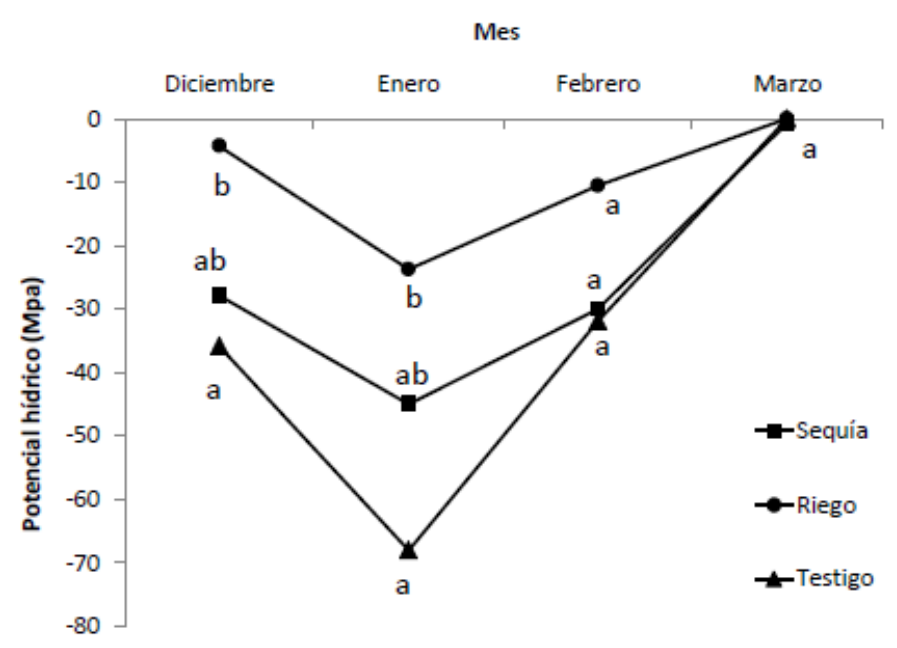

Figura 3. Potencial hídrico (MPa) del suelo de los tratamientos sequía, testigo y riego.

$\underline{\text { Cobertura de las formas de vida }}$

Las gramíneas cortas fueron dominantes con una cobertura media de $54.1 \%$ y los coirones aportaron en promedio $33.8 \%$ de cobertura. En el tratamiento riego se registró un mayor porcentaje de coirones $(38,7 \%)$ mientras que en el tratamiento sequía las gramíneas cortas mostraron mayor cobertura $(57,56 \%)$ (Tabla 1$)$.

La sumatoria de estas dos formas de vida no corresponde al 100\% dado que el marco estaba compuesto, además, por subarbustos y suelo desnudo no considerados en este análisis.

\begin{tabular}{ccc}
\hline & \multicolumn{2}{c}{ Cobertura absoluta $(\%)$} \\
\hline & Coirones & $\begin{array}{c}\text { Gramíneas } \\
\text { bajas }\end{array}$ \\
\hline \hline Sequía & $25,58( \pm 7,79)$ & $57,56( \pm 3,79)$ \\
Testigo & $37,29( \pm 11,99)$ & $56,63( \pm 3,08)$ \\
Riego & $38,69( \pm 12,24)$ & $48,46( \pm 15,81)$ \\
\hline
\end{tabular}

Tabla 1. Cobertura (\%) de las gramíneas altas (coirones) y bajas para cada tratamiento, entre paréntesis figura el desvío estándar, $n=3$.

$\underline{\text { Productividad primaria aérea neta }}$

La productividad media de los coirones fue de $24,74 \mathrm{~g} / \mathrm{m}^{2}$ y la de las gramíneas cortas de $12,48 \mathrm{~g} / \mathrm{m}^{2}$.

Los coirones en los tratamiento con riego y testigo $\left(10,7 \mathrm{~g} / \mathrm{m}^{2}\right)$ tuvieron tres veces más biomasa que en sequía $\left(3,32 \mathrm{~g} / \mathrm{m}^{2}\right)$, sin embargo, estas diferencias no fueron significativas (Figura 4). En la productividad de gramíneas cortas tampoco hubo diferencias entre los tratamientos evaluados. 


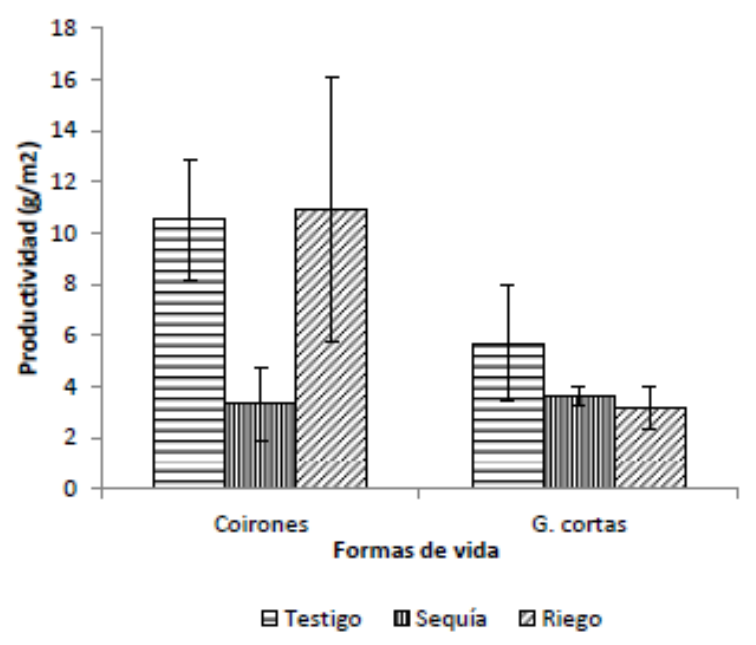

Figura 4. PPAN $\left(\mathrm{g} / \mathrm{m}^{2}\right)$ de gramíneas altas (coirones) y cortas para tres tratamientos diferentes y sus correspondientes errores estándares.

\section{DISCUSION}

El modelo de regresión propuesto por Jobbaggy et al., 2002, mostró que la estación de crecimiento comienza tardíamente en Potrok aike, ya que la temperatura del mes de julio fue menor a $0{ }^{\circ} \mathrm{C}$, esto dio como resultado que la vegetación empiece a crecer a mediados del mes de octubre. En cuanto a la humedad del suelo, el suelo mostró un corto periodo seco, que se dio principalmente en enero (Figura 3). Por lo tanto, la vegetación tuvo un periodo de crecimiento acotado entre octubre y enero. Aunque es probable, que haya habido crecimiento otoñal, ya que los meses de febrero y marzo del 2019 fueron húmedos (Figura 2) y este pico de crecimiento otoñal ha sido registrado para estos pastizales cuando las condiciones de humedad y temperatura son adecuadas (Paredes, 2011).

La simulación de sequía no produjo efectos sobre la productividad primaria aérea neta ni sobre la humedad del suelo. Se esperaba que este tratamiento intensifique la sequía en el verano o que prolongue el periodo seco en el tiempo. Sin embargo, ninguna de estas respuestas se observó en la parcela con interceptores de lluvia. Es posible que se requiere de un periodo más largo desde la instalación del interceptor de lluvia para generar un efecto real sobre la humedad del suelo. Además, las condiciones meteorológicas húmedas de fines de verano (febrero y marzo) que aportaron más de $75 \mathrm{~mm}$, generaron un pulso de humedad que recargó el perfil en todos los tratamientos.

El tratamiento de riego mantuvo el suelo más húmedo durante el verano. Aunque estas diferencias no fueron suficientes para generar cambios a nivel de PPAN de las formas de vida. En general, la respuesta en la vegetación a los pulsos de agua se manifiesta de manera jerárquica, desde niveles celulares hasta niveles ecosistémicos (Schwinning et al., 2004). Es probable que la diferencia de humedad de suelo generada en el mes de enero por los tratamientos impuestos en la EMS no haya sido suficiente para mostrar cambios a niveles de PPAN. Es probable que se requiera un periodo mayor para evaluar los cambios a esta escala. 


\section{CONCLUSIONES}

El periodo de crecimiento para la vegetación fue acotado. Inició en octubre, por las bajas temperaturas del mes de julio y finalizó en enero por déficit hídrico en suelo.

El tratamiento de riego tuvo un efecto sobre el potencial hídrico del suelo, pero la sequía no se evidenció en el tratamiento de intercepción de lluvia.

Las lluvias de fines de verano recargaron el perfil del suelo muy tempranamente en la estación cálida.

Los coirones tuvieron el doble de la PPAN $\left(24,74 \mathrm{~g} / \mathrm{m}^{2}\right)$ que las gramíneas cortas $\left(12,48 \mathrm{~g} / \mathrm{m}^{2}\right)$.

Las diferencias de humedad en suelo no fueron suficientes para tener un efecto a nivel de PPAN.

\section{BIBLIOGRAFIA}

FERRANTE, D. (2011). Distribución del agua en el suelo y su relación con la estructura radical y producción de biomasa de tres tipos funcionales, en un pastizal de la Estepa Magallánica Seca, Santa Cruz MS Thesis, Universidad de Buenos Aires, Buenos Aires, Argentina.

FERRANTE, D; OLIVA, G \& FERNÁNDEZ, R. (2014). Soil water dynamics, root systems, and plant responses in a semiarid grassland of Southern Patagonia. Journal of Arid Environments 104 (2014) 52e58. https://doi.org/10.1016/j.jaridenv.2014.01.009

JOBBAGY, E; SALA, O. E. \& PARUELO, J. (2002). Patterns and controls of primary production in the Patagonian steppe: a remote sensing approach. Ecology 83(2):307319. https://doi.org/10.1890/0012-9658(2002)083[0307:PACOPP]2.0.CO;2

LEÓN, R; BRAN, D; COLLANTES, M; PARUELO, J y SORIANO, A. (1998). Grandes unidades de vegetación de la Patagonia extra andina. Ecología Austral 8:125-144.

Noy-Meir. (1973). Desert ecosystems: environment and producers. Annual Review of Ecology and Systematics 4:25-51. https://doi.org/10.1146/annurev.es.04.110173.000325

OLIVA, G; GONZÁLEZ, L; RIAL, P y LIVRAGHI, E. (2001). El ambiente en la Patagonia Austral. Pages 40-45 in P. Borrelli y G. Oliva, editors. Ganadería ovina sustentable en la Patagonia Austral. Tecnología de Manejo extensivo. ErreGé \& Asociados, Buenos Aires.

PAREDES, P. (2011). Caracterización funcional de la Estepa Magallánica y su transición a Matorral de Mata Negra (Patagonia Austral) a partir de imágenes de resolución espacial intermedia. Tesis para optar al grado de Magister de la UBA, Universidad de Buenos Aires.

YAHDJIAN, L \& SALA, O. E. (2002). A rain shelter design for intercepting different amounts of rainfall. Ecology 133:95-101. https://doi.org/10.1007/s00442-002-1024-3

YAHDJIAN, L., \& SALA, O. E. (2006). Vegetation structure constrains primary production response to water availability in the Patagonian Steppe. Ecology 87 (4): 952962. https://doi.org/10.1890/0012-9658(2006)87[952:VSCPPR]2.0.CO;2

YAHDJIAN, L. y SALA, O. E. (2011) El futuro de los pastizales sudamericanos Interciencia, vol. 36, núm. 2, febrero, 2011, pp. 153-158. Asociación Interciencia Caracas, Venezuela.

SALA, O. E.; PARTON, W. J.; JOYCE, L. A. \& LAUENROTH, W. K. (1988). Primary production of the central grassland region of the United States. Ecology 69:40-45. https://doi.org/10.2307/1943158 
SALA, O. E.; LAUENROTH, W. K., \& GOLLUSCIO, R. A. (1997). Plant functional types in temperate semi-arid regions. Plant functional types: their relevance to ecosystem properties and global change, 217-233.

SALA, O. E. \& AUSTIN, A. (2000). Methods of Estimating Aboveground Net Primary Productivity. Page 421 in Sala O, Jackson R, Mooney H, y R. Howarth, editors. Methods in ecosystem science. Springer. https://doi.org/10.1007/978-1-4612-1224-9_3

SCHWINNING, S. \& SALA, O. E. (2004). Hierarchy of responses to resource pulses in arid and semi-arid ecosystems. Oecologia, 141(2), 211-220. https://doi.org/10.1007/s00442-004-1520-8 
ANEXOS

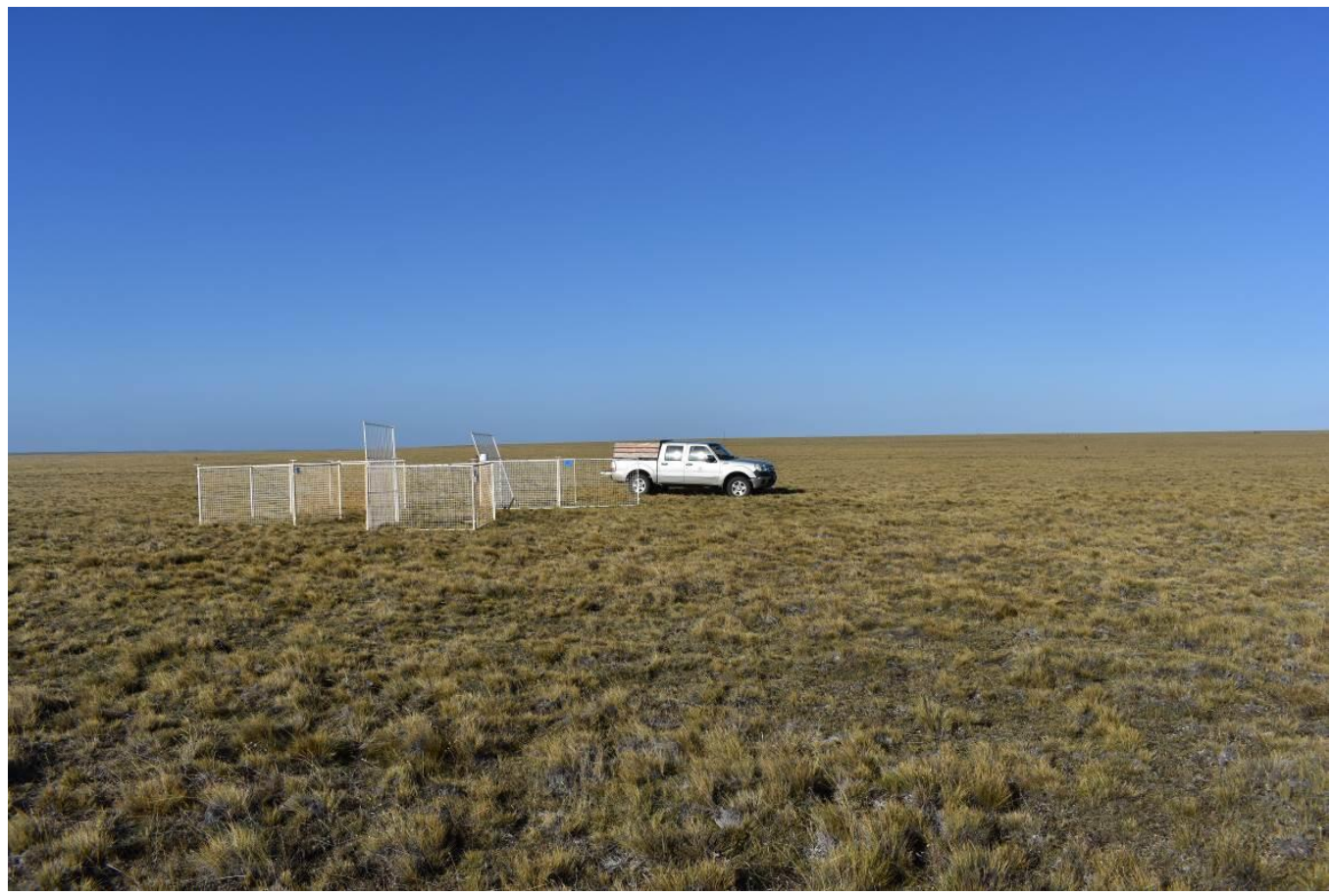

Cuatro de las parcelas del ensayo en un pastizal del campo experimental Potrok Aike.

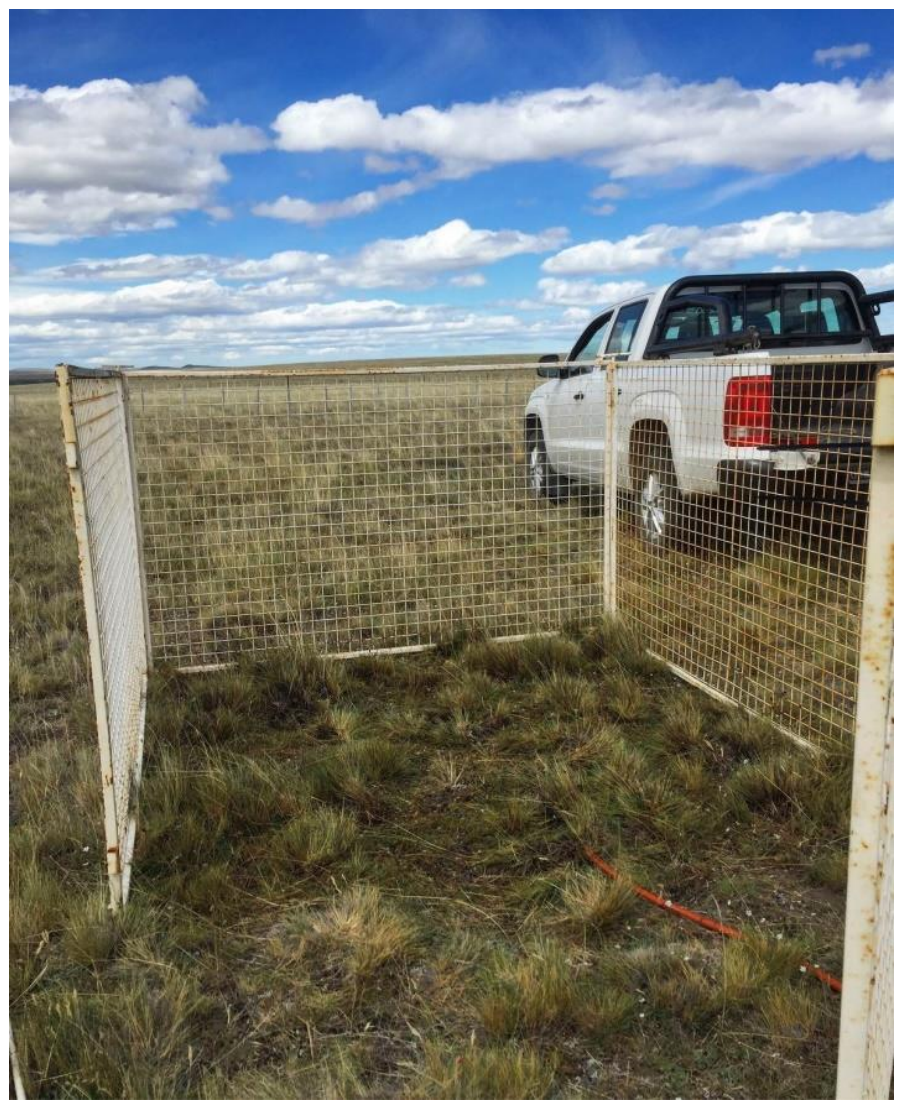

Parcela con sensor de potencial hídrico y temperatura del suelo, con registro por hora. 


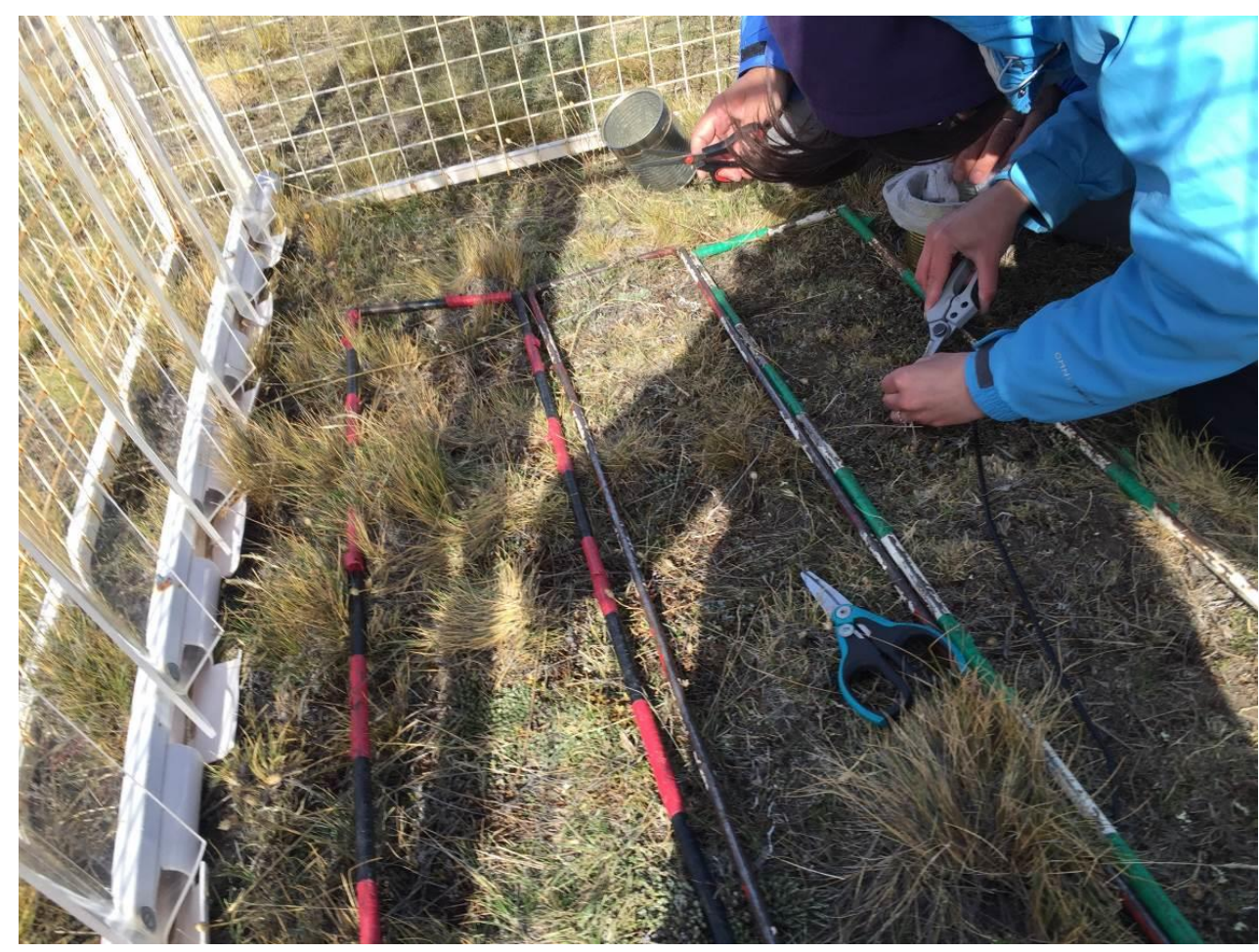

Cosecha de biomasa con marcos rectangulares de $0,2 \mathrm{~m}^{2}$. 